PALEO

Revue d'archéologie préhistorique

$27 \mid 2016$

Varia

\title{
Paléohistoire d'un crâne de Bison aurignacien à Régismont-le-Haut (Hérault, France) : de sa nature à sa valeur
}

Paleohistory of an aurignacian bison skull from Régismont-le-Haut (Hérault, France) : from nature to value

Jean-Philip Brugal

\section{OpenEdition \\ Journals}

Édition électronique

URL : http://journals.openedition.org/paleo/3176

DOI : $10.4000 /$ paleo.3176

ISSN : 2101-0420

Éditeur

SAMRA

Édition imprimée

Date de publication : 30 décembre 2016

Pagination : 62-82

ISSN : $1145-3370$

Référence électronique

Jean-Philip Brugal, « Paléohistoire d'un crâne de Bison aurignacien à Régismont-le-Haut (Hérault,

France) : de sa nature à sa valeur », PALEO [En ligne], 27 | 2016, mis en ligne le 01 juin 2018, consulté le 07 juillet 2020. URL : http://journals.openedition.org/paleo/3176 ; DOI : https://doi.org/10.4000/paleo. 3176

\section{(c) $($ () $\odot$}

PALEO est mis à disposition selon les termes de la licence Creative Commons Attribution - Pas d'Utilisation Commerciale - Pas de Modification 4.0 International. 


\title{
Paléohistoire d'un crâne de Bison aurignacien à Régismont-le-Haut (Hérault, France) : de sa nature à sa valeur
}

\author{
Jean-Philip BRUGAL (a)
}

Résumé : La découverte d'un crâne (et son atlas) de Bison dans le campement aurignacien de Régismont-le-Haut (Hérault) est assez inattendue dans ce contexte de plein air où les vestiges osseux sont mal conservés. Des approches complémentaires en paléontologie, taphonomie et archéozoologie permettent de retracer l'histoire (la paléohistoire) de cet animal et de discuter à la fois de sa position taxinomique mais aussi de sa valeur (économique, symbolique).

Mots-clés : Bison, France, Eurasie, taxinomie, taphonomie, archéozoologie.

Abstract: Paleohistory of an aurignacian bison skull from Régismont-le-Haut (Hérault, France): from nature to value. The discovery of a Bison skull (with its atlas) in the Aurignacian site of Régismont-le-Haut (Hérault) is relatively surprising in this openair context where faunal remains are not well preserved. Complementary studies in paleontology, taphonomy and zooarchaeology allow to reconstruct the history (paleohistory) of this specimen and to discuss on its taxonomical position as well as about its meaning or value (economic, symbolic).

Key-words: Bison, France, Eurasia, Taxonomy, Taphonomy, Zooarchaeology.

Régismont-le-Haut désigne un campement du Paléolithique supérieur ancien (Aurignacien), un des rares connus en plein air en Europe, tout du moins ayant bénéficié d'une telle d'intégrité. Localisé dans les plaines du Languedoc, il a livré un important matériel archéologique (vestiges lithiques, matières colorantes, coquillages, etc.) associé à de nombreuses traces d'occupations, comptant notamment de nombreuses structures de combustion, le tout réparti sur une surface de l'ordre de $600 \mathrm{~m}^{2}$ dont la moitié environ de fouillée (Bon, Mensan et al. 2007, 2008 ; Anderson et al. à par.). Les matières minérales sont bien préservées bien que les restes osseux soient généralement mal conservés, à l'exception inattendue d'un crâne de bison. Celui-ci fait l'objet de cette contribution et sert de support pour aborder à la fois son contexte taphonomique et sa signification anthropologique, mais aussi le statut taxinomique des grands Bovidés de la fin du Pléistocène supérieur.

\section{Contexte de la découverte}

Le site de Régismont-le-Haut (Poilhes, Hérault) fait l'objet de fouilles programmées dirigées par F. Bon (Université de Toulouse Jean Jaurès) et R. Mensan depuis 2000 (Bon, Mensan et al. 2007, 2008). II s'agit d'un site de plein air localisé sur les contreforts de la colline d'Ensérune (d'âge Miocène), au cœur de la plaine du Biterrois, qui livre une industrie d'un Aurignacien difficile de fixer précisément dans le temps (Aurignacien récent ?). Ce site recèle une ou 
plusieurs occupations (saisonnières ?) d'un groupe de chasseurs-collecteurs, associée à de nombreuses structures, en particulier foyères ${ }^{1}$. Le sédiment est formé de colluvions sablo-limoneuses, en relation avec un chenal, qui se prêtent mal à la conservation de restes organiques (ex. ossement et dent). En effet, la plupart des fragments osseux recueillis sont réduits à l'état de " fantômes ", hormis les vestiges carbonisés de petite taille ou bien ceux appartenant sans doute à des accumulations plus denses, comme à proximité des foyers XII-XVI (secteur S56). II s'agit alors de microfragments dentaires non identifiables et de petits fragments diaphysaires dont les épaisseurs corticales correspondraient à un grand ongulé (taille bison ou cheval).

Cependant, en 2005, dans le secteur S72, un crâne de bison fut découvert (fig.1), avec à proximité immédiate sa première vertèbre cervicale (atlas), suivi par la suite d'un coxal, mal conservé, du même genre et provenant du même secteur. À l'évidence, la masse osseuse représentée par cette pièce pourrait en partie expliquer une meilleure conservation que celle observée sur d'autres restes osseux de plus petite taille et très dispersés. Plusieurs fragments dentaires ainsi que deux molaires supérieures gauches, avaient été trouvés avant la découverte du crâne lui-même dans la même zone. Ce crâne se trouvait à trois mètres d'un foyer (F. XIV), environné de quelques vestiges lithiques et de colorants. Parmi ces pièces lithiques figure un débitage de quartz dont l'un des éclats a été découvert à proximité immédiate du crâne ; l'hypothèse d'un instrument de boucherie a été posée, sans toutefois avoir pu être validée par l'étude fonctionnelle (par M. Araujo) en l'absence de traces diagnostiques.

Ce sont donc des pièces de dimensions importantes qui semblent seules avoir été préservées, et le matériel dentaire lui-même est pratiquement absent, témoignant de l'agressivité du milieu sédimentaire. L'étude géoarchéologique cependant, indique que les traces d'occupations humaines bénéficient d'une bonne conservation structurelle, au sein d'un replat accompagné d'érosion marginale (Bon, Mensan et al. 2007, 2008), ce qui suggère une relative absence de grand déplacement du matériel. L'intégrité des structures de combustions confirme cette hypothèse qui constitue un aspect important pour l'interprétation taphonomique du crâne de bison (cf. infra).

Suite à la découverte du crâne, une première intervention sur le terrain en septembre 2005, sur l'invitation de F. Bon et $\mathrm{S}$. Costamagno, m'avait permis à la fois d'identifier le spécimen au genre Bison et d'effectuer des premières observations et mesures. Le crâne était retourné montrant sa face basale, légèrement en oblique, avec l'atlas proche. Cette vertèbre, en mauvais état de conservation, ne se prête pas à une étude morphométrique. Par la suite, le crâne a été soigneusement plâtré, apporté au laboratoire de Toulouse puis restauré (Kuntz 2006), tout en étant retourné alors et présentant ainsi sa face dorsale (fig. 2). Cette nouvelle orientation a permis de reprendre une série de mesures en juin 2006 qui complètent ou précisent les premières mensurations. Celles-ci se recoupent parfois et ne coïncident pas toujours avec les mêmes dimensions prises en 2005 ; elles ont été alors moyennées pour certaines. Quoi qu'il en soit, et considérant l'état général de la pièce, les mesures ne sont parfois qu'estimées, ce qui est souvent le cas des bisons fossiles découverts et publiés, provenant de gisements européens.

\section{Description et première interprétation}

Le crâne est relativement complet ; il manque cependant la partie dorsale antérieure (bas du frontal, nasaux et partie antérieure de l'os incisif ou prémaxillaire), les pointes des chevilles osseuses, les dents supérieures (des petits fragments d'émail de molaires ont été retrouvés près du maxillaire, donc légèrement au-dessus du crâne au moment de la découverte) ; la face nucale est altérée avec un basioccipital mal préservé et des condyles occipitaux abrasés ainsi que la crête (ou saillie) nucale. La partie antérieure manquante est la plus fragile et correspond à la région qui conserve le plus longtemps des sutures ouvertes (nasal, bas du frontal), même sur des animaux adultes (cf. fig. 3 pour comparer avec des sujets âgés de bison européen). L'ensemble est cependant en relatif bon état, non comprimé ou déformé, avec des surfaces osseuses variables; de rares fissures (sur l'os frontal qui paraît épais) témoignent d'une légère compaction sédimentaire. Les sutures crâniennes ne sont pas visibles, toutes fermées, ce qui indique à la fois l'état adulte de l'animal et pourrait expliquer également sa relative bonne conservation globale. Du sédiment est présent dans la cavité cérébrale. La taille générale de cette pièce et son aspect massif suggèrent une attribution à un mâle (cf. infra) : en comparaison le crâne des femelles est souvent plus fin et fragile avec des chevilles osseuses moins compactes (les sutures se ferment lorsque la fonction de reproduction est terminée, soit vers 13-15 ans, et jusqu'à 20 ans). Chez les mâles, ces sutures se ferment vers 6-7 ans (Flerow 1979 ; Vasiliev 2008) et c'est pourquoi il est relativement plus fréquent de découvrir des crânes de bison mâle que de bison femelle, en rapport avec cette plus grande robustesse, notamment en contexte alluvial (Vasiliev 2008 : sites alluviaux de Sibérie occidentale).

D'un point de vue taphonomique on peut relever certains points : la présence de l'atlas associé au crâne indique une connexion et ces deux éléments sont restés ensemble tout

(1) "Même s'il n'a pas encore été possible d'en apporter la preuve au travers notamment de remontages lithiques, l'hypothèse que ce site corresponde à une seule phase d'installation, formant alors un vaste campement, semble devoir primer sur celle de la répétition de courtes installations ; nous verrons d'ailleurs que ce crâne apporte tout à la fois des indices d'un recouvrement rapide comprimant l'intervalle de temps nécessaire au scellement des traces constituant ce site, tandis que la quantité de viande livrée par cet animal permet d'envisager qu'un groupe significatif ait pu ainsi être alimenté - même si cela reste impossible à prouver " (F. Bon, comm.pers.) 


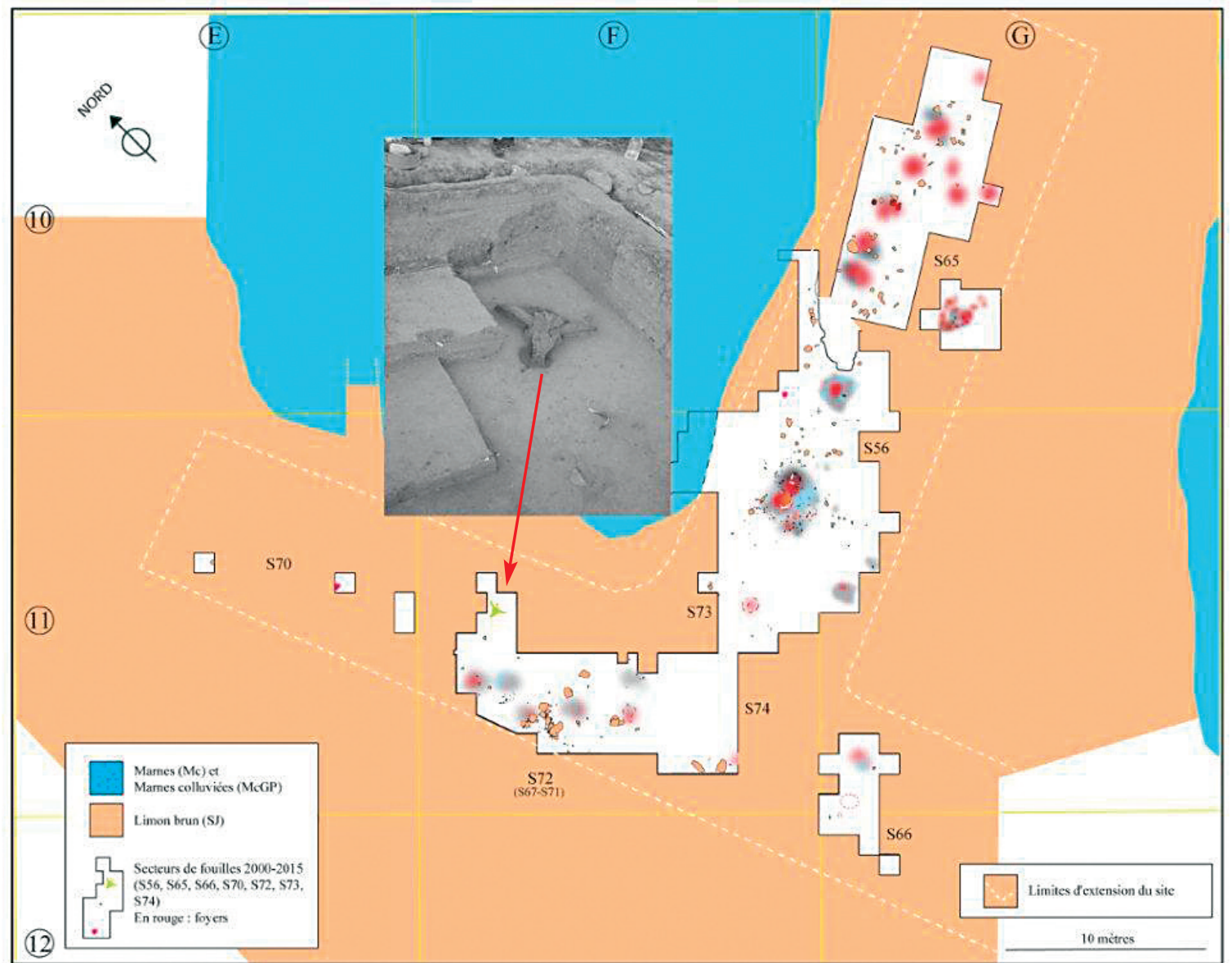

Figure 1 - Vue générale du site de Régismont et localisation du crâne de bison (en vert), vue in situ (septembre 2005 : vue basale/ventrale) (photos F. Bon).

Figure1 - General map of the site of Régismont and location of the bison skull (in green), view in situ (September, 2005: ventral view) (photos F. Bon).

au long de leur « histoire », avant, pendant et après enfouissement ; ils ne sont pas toutefois en connexion stricte et on peut penser qu'une exposition subaérienne a fait lâcher les ligaments et que l'atlas est tombé, légèrement éloigné du crâne, avant leur dépôt définitif. II est à cet égard intéressant de noter qu'une couverture d'environ cinq centimètres de sédiment s'était déposée avant le détachement de la vertèbre ; sa base est donc sensiblement plus haute que celle du crâne, ce qui corrobore les hypothèses émises en faveur d'un enfouissement rapide et naturel (i.e., ce ne sont pas les hommes qui auraient enfoui ce crâne par exemple). Toutefois, la quasi absence de dents est révélatrice d'une exposition suffisamment conséquente pour permettre à la plupart d'entre elles de se déchausser ou de se fissurer, et de tomber du crâne, avant de disparaître par digestion sédimentaire (physico-chimique, cycle cryogénique). Quoi qu'il en soit, la bonne préservation générale du crâne permet d'avancer que si l'exposition a été réelle, de l'ordre de quelques années ou saisons, l'enfouissement de celuici a été assez rapide, assurant au final cette bonne conservation. Si cela n'avait pas été le cas, les parties les plus fragiles, souvent exposées (en relation avec la hauteur de la pièce), auraient disparu, en particulier les chevilles osseuses spongieuses. Ceci a été observé dans des sites nord-américains paléoindiens où les crânes ont souvent la région supérieure (avec les « cornes ») souvent décapitée, ou oblitérée, suite à un enfouissement relativement lent (Todd et Rapson 1999 : fig.4, p. 490) et une longue exposition. II est alors possible d'avancer que l'ensevelissement fait suite à un (ou des) moment(s) rapproché(s), à caractère évènementiel, de type colluvionnement et un dépôt sédimentaire rapide et assez épais. De plus, et suite à l'observation visuelle des parties exposées (sur le terrain et en labo), et malgré une certaine altération des surfaces (sous la forme d'encroûtement pelliculaire), nous n'avons pas relevé de traces particulières, notamment d'origine anthropique (stries de découpes ou de radicelles). Une zone d'enfoncement est observable entre les chevilles sur la surface frontale gauche, d'origine vraisemblablement naturelle (sédimentaire), et aucune fissuration n'apparaît sur ces surfaces. 

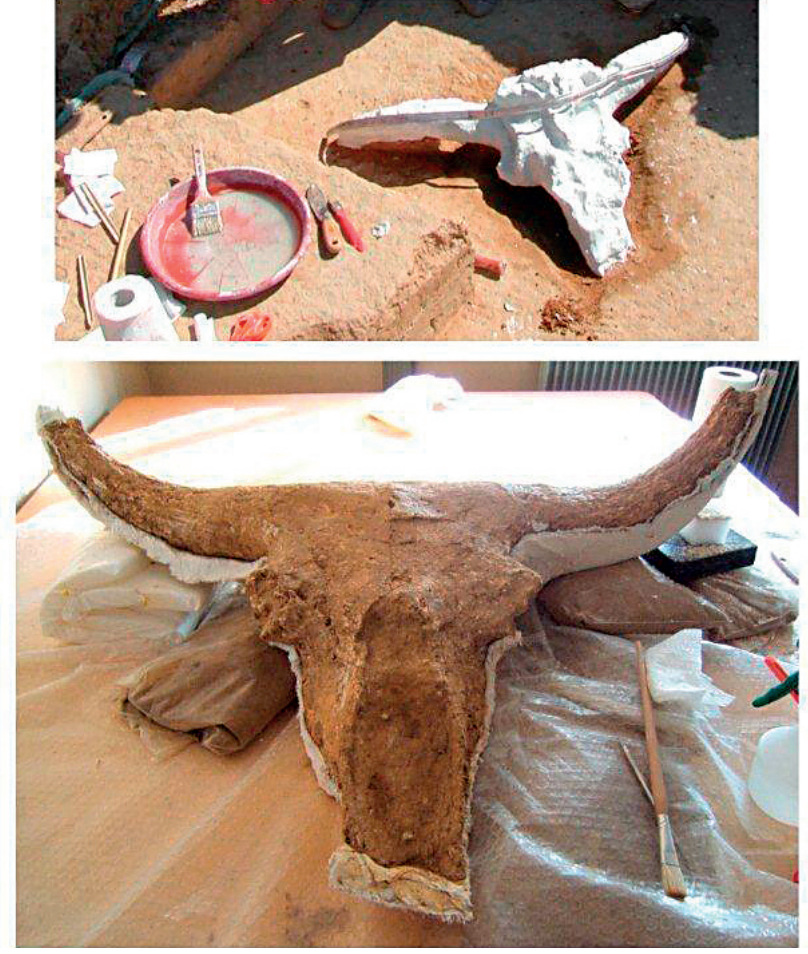

Figure 2 - Dégagement et restauration (Juin 2006 : vue frontale) (photos F. Bon).

Figure 2 - Excavation and restoration (June 2006, frontal view) (photos F. Bon).

L'origine de cette pièce reste à élucider et, de fait, deux hypothèses, en partie antagonistes, peuvent être proposées. La première suggère que le crâne de ce bison correspond à un individu mâle chassé et tué par les Aurignaciens. La seconde le considère comme un vestige collecté sur le lieu même du campement ou très proche de celui-ci.

Dans le cadre de la première de ces hypothèses, la tête de l'animal aurait été détachée (entre l'atlas et l'axis) et la mandibule séparée (avec prélèvement de la joue et de la langue (?), parties très nutritives). En revanche, la cervelle ne paraît pas avoir été récupérée, si l'on en juge par le caractère intact de la cavité céphalique, la présence de l'atlas condamnant de son côté l'accès par le foramen occipital. Or, outre l'intérêt alimentaire de la cervelle, son usage lors de certaines étapes du traitement des peaux ne trouve ici aucune confirmation alors que cette activité est fortement marquée à Régismont, par le type d'outillages lithiques et les traces qu'ils portent (Bon et al. soumis). Cependant, dans le contexte de chasse au bison, si un seul individu peut être abattu de manière unique, voire occasionnelle, d'autres exemples illustrent des tueries plus nombreuses, ou plus récurrentes sur cette espèce grégaire (chasse collective où plusieurs individus sont abattus, ou abattage régulier au cours du temps) (Brugal et al. 1999). La tête d'un unique individu, tête qui deviendra crâne, aurait donc été mise de côté intentionnellement, éventuellement pour un autre usage, délaissant la ressource encore exploitable.

Les crânes sont en effet, par nature, des trophées appréciés des chasseurs et il n'est pas rare, voire fréquent, que ceux-ci soient conservés, exhibés et utilisés, parfois décorés dans les sociétés traditionnelles ; les exemples ethnographiques sont nombreux, notamment chez les sociétés à bison des indiens nord-américains (e.g. GarraitBourrier 2006). Les exemples archéologiques paléoindiens sont également nombreux : crâne peint de bison (Bement 2003 ; et voir Nicholson et Nicholson 2007), structure de crânes (Frison 1971 ; Reher et Frison 1980).

Le crâne de Régismont correspond à un individu de forte taille, adulte mâle en pleine maturité physiologique et force, ce qui représente une proie de qualité pas toujours facile à chasser et tuer. Dans ce cadre, nous suggérons que ce crâne (avec son atlas) ait pu être suspendu, ou en tout cas placé en position relevée, droite, peut être planté au bout d'un piquet en bois qui s'est désagrégé avec le temps et a fait chuter l'ensemble, dispersant dents et atlas. Cette pratique, tout à fait plausible chez les Hommes anatomiquement modernes, aurait une fonction symbolique forte, pour la première fois signalée pour les sociétés du Paléolithique supérieur ancien.

La deuxième origine possible de cette belle pièce, consiste à penser qu'elle aurait été collectée autour du site, voire même ramassée sur le site même. Le crâne aurait alors été trouvé déjà décharné et sans la peau, la mandibule dissociée, une partie des dents déjà déchaussée, la partie céphalique intacte et sans traces anthropiques apparentes. La présence de l'atlas est liée à l'existence d'une très forte liaison ligamentaire entre crâne et première vertèbre cervicale (Hill 1979) et permet d'inférer en partie l'intervalle de temps entre la mort et l'enfouissement (Hill et Behrensmeyer 1984). Dans notre cas, on estime un temps d'exposition entre 2 et 4 ans, le crâne commençant à sécher, jusqu'à la perte finale de l'atlas (pas loin du crâne) et un recouvrement sédimentaire rapide. La présence de ce crâne en ce lieu aurait pu représenter un signe positif de l'existence de cette espèce grégaire sur ce territoire et, s'il ne désigne pas un réel augure, sa collecte et son utilisation à des fins autres qu'alimentaires sont envisageables. La pratique probablement symbolique, à base cynégétique, évoquée plus haut prendrait encore une grande réalité dans ce contexte. Cependant, devant le bon état général de cette pièce, sans trop de trace d'exfoliation ou de weathering des surfaces, l'hypothèse d'une simple collecte nous semble néanmoins plus incertaine et c'est plutôt la première hypothèse que nous retiendrons, celle d'une portion particulière prélevée sur un animal abattu et exhibée.

\section{Étude paléontologique et taxinomie}

Le squelette céphalique de Bison se distingue de celui de Bos sur les points suivants, correspondant d'ailleurs aux grandes tendances évolutives des Bovini (Gromova 1932, 1935 ; Pilgrim 1947 ; Bohlken 1958 ; Geraads 1992 ; Pfeiffer 1999) : chez le bison on assiste à un élargissement 


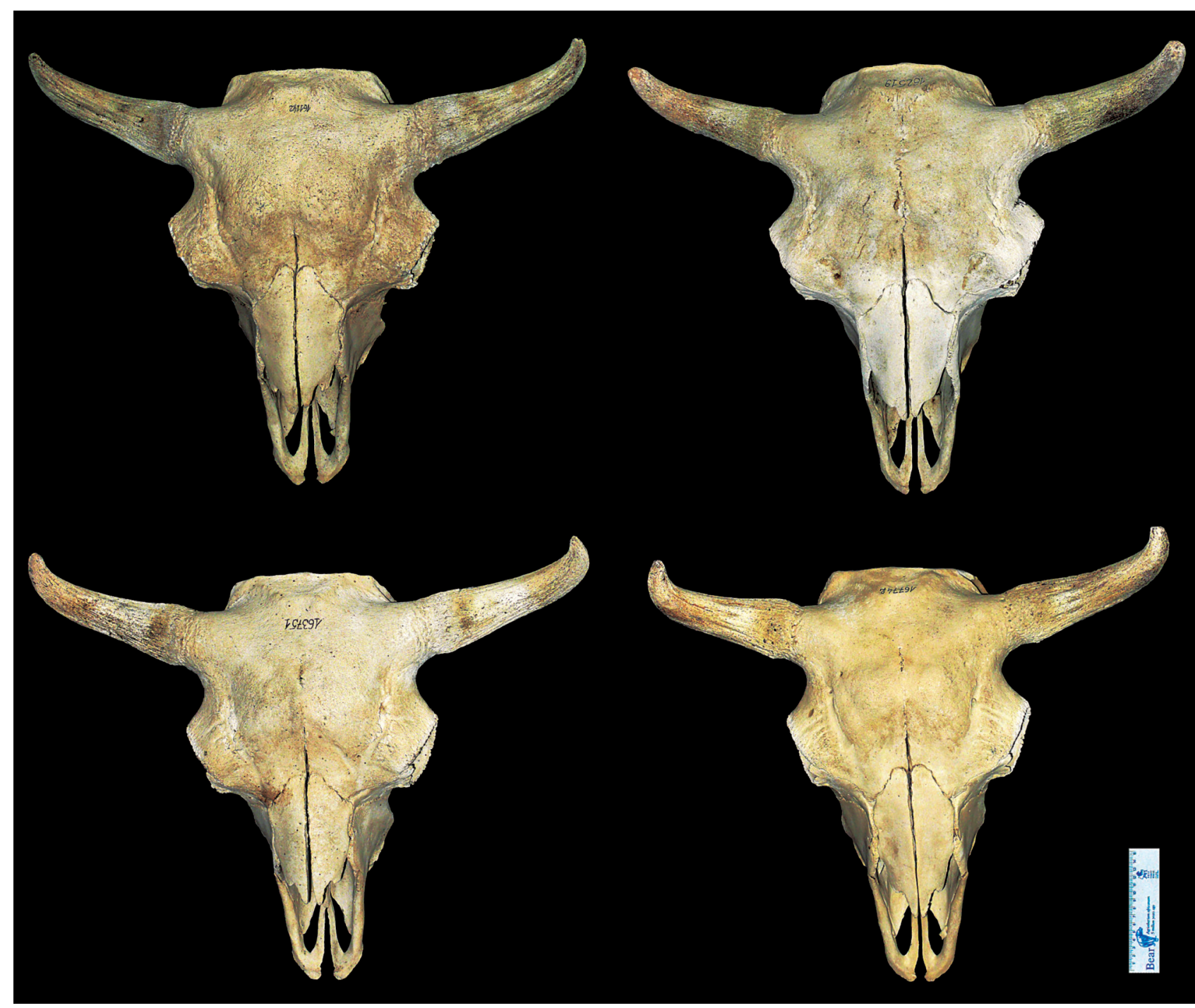

Figure 3 - Photos de Bison bonasus mâle actuel (vue frontale/dorsale) (cf. tabl. 4 ; nº MRS, Pologne : haut G : 161142, D : 162519 ; bas G: 163751, D: 167742 - photos Ph. Fosse).

Figure 3 - Photos of modern mâle Bison bonasus (frontal view) (cf. tabl.4; $n^{\circ}$ MRS, Poland: haut L: 161142, R: 162519; bas L: 163751, R: $167742-$ photos Ph. Fosse).

important de la surface frontale sans refoulement total de la région pariétale vers la surface occipitale (caractère primitif) ; le frontal est relativement plus court que chez Bos ; le pariétal participe pro parte à la formation de la voûte crânienne chez le bison ; l'occipital est relativement plus large et plus bas et la protubérance occipitale est bien développée en arrière des chevilles osseuses ; l'ouverture postérieure de la fosse temporale est plus grande ; les orbites, plus proéminentes, sont dirigées dans la plupart des cas vers l'avant et le haut. La morphologie d'ensemble du crâne chez l'aurochs (et ses dérivés) est plus longue et étroite que chez les bisons qui ont un crâne large et plus compact. Les chevilles osseuses de bison montrent une section plus ou moins (sub)triangulaire à ovale (diamètre horizontal supérieur au diamètre vertical à la base) et se développent tout d'abord légèrement vers le bas et l'arrière puis plus nettement vers le haut, avec les pointes plus ou moins inclinées vers l'arrière (faible torsion) ; la courbure est non hélicoïdale et, enfin, chez les sujet âgés, la surface des chevilles est parcourue de cannelures longitudinales accusées.

Au niveau spécifique, l'architecture crânienne est comparable entre les bisons fossiles, priscus et schoetensacki (Flerow 1975, 1976 ; Sala 1987) et actuels bonasus et bison (Empel 1962 ; McDonald 1981). La largeur du frontal et sa courbure changent en fonction de la dimension des chevilles osseuses. Celles-ci varient 
fortement à l'intérieur d'une espèce dans la forme, la longueur et la courbure en rapport avec le sexe et l'âge des individus. Cependant, on peut avancer quelques remarques sur les cornes : chez $B$. priscus, les chevilles sont orientées vers le bas à leur départ du crâne, elles se courbent vers les côtés ou vers 1'arrière et dépassent le crâne, elles sont rarement spiralées ; chez B. schoetensacki, les chevilles osseuses sont plus courtes et massives, elles se rétrécissent de la base au sommet, la base est aplatie et la section devient plus circulaire vers les $2 / 3$ distal, la courbure est faible en avant dans le $1 / 3$ proximal puis se courbe vers le haut et dans le $1 / 3$ final vers l'arrière, les chevilles sont relativement rectiligne (Brugal 1994-95). Les espèces actuelles sont similaires au bison des steppes, $B$. priscus, avec quelques différences à la fois dans les chevilles osseuses plus courtes et une relative moindre taille corporelle, et aussi dans le port de tête : en position plus basse chez bison (grazer) que chez bonasus (mixed-feeder à browser).

Le crâne de Régismont (fig. 2) est typique de Bison, comparable à la lignée priscus ou assimilés (cf. infra). Ses chevilles osseuses sont assez imposantes, dirigées latéralement (angle d'environ $80^{\circ}$ avec le plan sagittal) et légèrement vers l'arrière et le bas avant de recourber et se redresser vers le haut puis elles semblent partir vers l'arrière au niveau des pointes, avec une très faible torsion. Les chevilles des femelles, moins lourdes, se dirigent latéralement tout en restant plus rectiligne sans pencher vers le bas (Prat et al. 2003). La section des chevilles de Régismont est sub-ovalaire à la base alors qu'elle est plus circulaire par exemple chez B. priscus d'Habarra (Prat et al. 2003 : fig. 25-26) ou de Gandil (Griggo 2014). La section est variable (subcirculaire, subovalaire et même - plus rarement - subtriangulaire) sur des bisons européens actuels (Brugal, obs.pers.). II semble également qu'il y a peu ou pas de cannelures (face inférieure), caractère en relation avec l'âge. La protubérance entre les chevilles, au niveau du frontal (vue nucale) est peu marquée. Les orbites sont fortes, tubulaires et assez proéminentes, dirigées vers le bas. La morphologie générale est donc similaire à celle des bisons modernes européens (fig. 3) bien que ceux-ci affichent des chevilles osseuses raccourcies et des bosses frontales plus accentuées.

II existe un fort dimorphisme sexuel chez les bovinés, la femelle étant de plus petite taille que le mâle. De nombreux travaux (e.g. Gromova 1932, 1935 ; Flerow 1979 ; McDonald 1981) montrent que les femelles ont des chevilles osseuses considérablement plus courtes, différentes parfois dans la courbure, que les mâles. De plus, il existe également une forte variabilité individuelle, en relation avec l'âge des individus et vraisemblablement avec les catégories et qualités nutritives de l'alimentation. Ces observations et ce très fort dimorphisme entre sexes, doivent être absolument considérés lorsqu'on travaille sur ce groupe de grands Bovidés, tant au niveau crânien qu'appendiculaire d'ailleurs (e.g. Brugal et Fosse 2005).

Cette variabilité morphologique, phénomène d'adaptation à des conditions environnementales changeantes, se reflète dans la variation métrique ; les mesures effectuées sur des sujets mâles restent plus grandes que celles faites sur des sujets femelles. En général les crânes fossiles sont des spécimens peu fréquents dans les collections car plus sensibles aux altérations et destructions; sur le plan morphométrique, les dimensions sont souvent estimées, donc approximatives. De plus, dans le cas de Régismont, certaines mesures n'ont pu être faites en raison de l'impossibilité de manipuler cette pièce imposante (et aussi en raison de zones altérées). Rappelons que le crâne repose désormais sur la face inférieure (exposant sa face frontale) dans une coque plâtrée (fig. 2) : en particulier, des mesures de hauteur par exemple, ou de longueur basale, n'ont pas pu être prises et d'autres n'ont pu qu'être appréciées (notées avec un e. = environ dans les tableaux).

Les bisons ont une vaste distribution géographique et chronologique, couvrant toute l'Eurasie et l'Amérique du Nord. Nous avons toutefois limité notre comparaison biométrique à l'Eurasie, dans un double espace taxinomique et chronologique somme toute limité. Les tableaux 1-3 a-b réunissent quelques données sur les dimensions crâniennes concernant des sites du Pléistocène supérieur d'Europe de l'Ouest (France, Belgique), mais aussi au-delà, de Sibérie, avec des formes attribuées à $B$. priscus priscus auxquelles nous avons rajouté des données sur le bison européen $B$. bonasus, le Wisent (tabl. 4). Les bisons nordaméricains (priscus/crassicornis, antiquus, occidentalis, bison, athabascae...) n'ont pas été considérés ici. Ce tableau ne prétend pas être exhaustif : une première lecture permet de rendre compte de la disparité (i.e. manque) dans les jeux de données et, comme nous l'avions déjà signalé, des approximations des mesures ; il s'agit le plus souvent d'individus et découvertes isolées (hors les séries de Sibérie occidentale). Ces données limitent alors des analyses globales et les illustrations graphiques.

La seule longueur disponible pour le crâne de Régismont est la longueur condylo-basale (mesure 2) estimée à $495 \mathrm{~mm}$, inférieure à celle d'un mâle de $B$. priscus d'Habarra (A55-B1) estimée à $597 \mathrm{~mm}$. Par contre, cette dimension est proche de celle des mâles de $B$. bonasus : $(n=5)$ 480-520, moyenne de 507 $\pm 18,6$. Dans cet ensemble, le crâne de Riverenert (Ariège) affiche des valeurs les plus importantes de l'ensemble de notre échantillon. Cette pièce, un peu hors-norme, a été décrite comme $B$. priscus gigas (Crouzel, Manenc, Revel 1982) et il est certainement le plus ancien en âge, probablement du Pléistocène moyen.

L'examen des largeurs (I.) crâniennes est plus informatif : les mesures 33 et 32 (respectivement I. maximale au niveau des orbites et I. du frontal entre orbites et chevilles, tabl. 1) restent globalement comparables entre les espèces mais montre un dimorphisme sexuel plus accusé (fig. 4), les sujets mâles ayant des valeurs plus importantes. II existe un chevauchement entre les deux taxons, mais ce sont certains priscus de Sibérie qui ont les valeurs les plus fortes. La largeur minimale entre la base des chevilles (m.31) et la largeur minimale au-dessous des orbites 
(m.34) sont aussi plus grandes chez priscus (proche entre Régismont et les sites belges) que chez bonasus mâle, alors que la largeur à la limite Prémaxillaire-Nasal est similaire aux sujets modernes. Les dimensions des orbites du crâne de Régismont sont les plus fortes, mais peut-être s'agit-il de techniques de mesures légèrement différentes (prise de mesures à l'intérieur vs à l'extérieur des orbites). Les longueurs des chevilles osseuses (m.47) sont beaucoup plus importantes chez priscus que chez bonasus. De même, la distance entre les pointes (apex) est plus grande, en particulier chez les mâles priscus où elle avoisine et excède facilement $1 \mathrm{~m}$, ce qui est comparable à Régismont (hors le crâne de Riverenert avec ses $2 \mathrm{~m}$ d'envergure !) ; les mâles de bonasus ont des valeurs comprises entre 52 et $65 \mathrm{~cm}$ (presque moitié moins). Enfin, les dimensions des chevilles osseuses elles-mêmes sont relativement plus fortes chez priscus que chez bonasus (fig. 5) ; cependant les valeurs pour les femelles de la première espèce restent comparables à celles des mâles de la deuxième. La morphologie des sections est assez variable également, et Régismont, comme les sujets de Sibérie ou de Belgique, montre des circonférences assez aplaties dans le sens dorso-ventral, et présente une section plus ovalaire, caractère vraisemblablement plus masculin.

Pour résumer, la morphologie du crâne de Régismont désigne sans ambiguïté un bison mâle adulte. Si les dimensions crâniennes restent somme toute assez proches entre certains priscus du Pléistocène supérieur et des spécimens bonasus actuels, les chevilles osseuses, par contre, montrent un développement plus fort chez l'espèce fossile. Cette variation pourrait se retrouver dans les représentations pariétales (Fritz et Tosello, comm. pers.). Cependant, la prise en considération du sexe d'une part, et de l'âge d'autre part (âge ontogénique mais aussi chronologique), tend à compliquer ce schéma et met en avant l'existence d'une grande variabilité des spécimens. On constate ainsi une certaine proximité morphométrique entre les bisons du Pléistocène supérieur et ceux de l'Holocène. La taxinomie des grands bovidés (Bovinı), en particulier le genre Bison (ou sous-genre pour certains : Groves 1981 ; Wall, Davis, Read 1992 ; Hassanin, et al. 2013 ; Soubrier et al. 2016), les relations phylétiques entre espèces fossiles et modernes et leurs dynamiques évolutives, restent encore ambiguës et sujettes à débat. Ceci est pro parte en relation notamment avec la très grande distribution géographique et chronologique de ces taxons. Ils sont présents dans l'espace eurasiatique au moins depuis le Pléistocène inférieur. Plusieurs études moléculaires récentes (basées sur le nuADN et mtADN) ont permis de préciser quelques points tels que la paraphylie entre Bos et Bison, ou la monophylie de Bison, mais aussi de montrer une importante variabilité génétique, à partir des taxons modernes et fossiles (e.g., Hassanin et al. 2013 et biblio.). Ainsi, une autre forme de bison coexisterait - ou succéderait - au bison des steppes au Pléistocène supérieur, forme ou clade rapprochée du bison européen actuel (Cooper et al. 2015 ; Soubrier et al. 2016 ; Massilani et al. 2016). L'origine de B.bonasus serait une hybridation (introgression) entre le bison des steppes et l'aurochs avant 120 ky (Soubrier et al. 2016), ou même peut être plus anciennement (Massilani et al. 2016), en Europe centrale ; cette espèce serait présente en Europe de l'Ouest, de manière discontinue, au moins depuis $50 \mathrm{ka}$.

Ces données posent la question de la détermination taxinomique des bisons découverts dans les gisements ouest européens paléolithiques, de même que le type (espèce) de bison représenté dans l'art pariétal ou mobilier ; certains auteurs considèrent que deux formes ou espèces seraient présentes (Fritz et Tosello, com. pers. ; Spassov et Stoytchev 2003). Le sujet reste ouvert, et des approches morphofonctionnelles (2D, 3D) pourraient être plus développées, entre matériel fossile et moderne (voir par ex. Vasiliev 2008 sur les métacarpiens) afin de rendre compte de l'existence de taxons différents².

En fonction de ces données et paramètres, à quel taxon le crâne de Régismont-le-Haut se rapporte-t-il ? Sa morphologie permet une claire attribution à Bison - ou Bos (Bison) - et ses proportions crâniennes permettent de le regrouper avec les formes priscus-bonasus du Pléistocène supérieur et de l'Holocène. De plus, comme d'autres spécimens eurasiatiques rapprochés du bison des steppes $B$. priscus ssp., le crâne de Régismont a des chevilles osseuses de plus grande taille. C'est donc à cette espèce que nous identifierons le bison de Régismont, même avec quelque réserve : en effet, on pourrait envisager que les anciens $B$. bonasus présents vers la fin du Pléistocène possédaient également des cornes beaucoup plus importantes que les représentants actuels de cette espèce. Cette étude nous offre ainsi l'occasion de souligner que l'équation bison pléistocène (supérieur) égal $B$. priscus n'est peut-être plus si évidente. L'évolution des bisons au cours du Pléistocène est marquée par une diminution de la taille corporelle, avec des variations d'ordre éco-climatique (écotypes). Les populations de la fin du Pléistocène semblent en moyenne de même taille que les bisons européens actuels : sites de Willment' Pit ou Shropham en Angleterre (Brugal, 1999 : fig.3, sur les longueurs des métapodes) comme aussi à Siréjol, Habarra ou l'Arquet en France. Mais s'agit-il encore réellement de priscus? En effet, c'est le clade de bonasus qui a été reconnu dans le site en aven de l'Arquet dans le Gard, avec une séquence fossilifère datée entre 45 et 26 ka (Gamberi et al. 2011 ; Massilani et al. 2016).

(2) Alors que cet article était sous-presse, un papier sur un ADN fossile d'un seul os (Palacio et al. 2017), daté de 36 ky, ressuscitait un Bison schoetensacki, espèce disparaissant au début du Pléistocène moyen, et dont on ne connaît d'ailleurs pas le génome... ce qui nous paraît assez étonnant. 

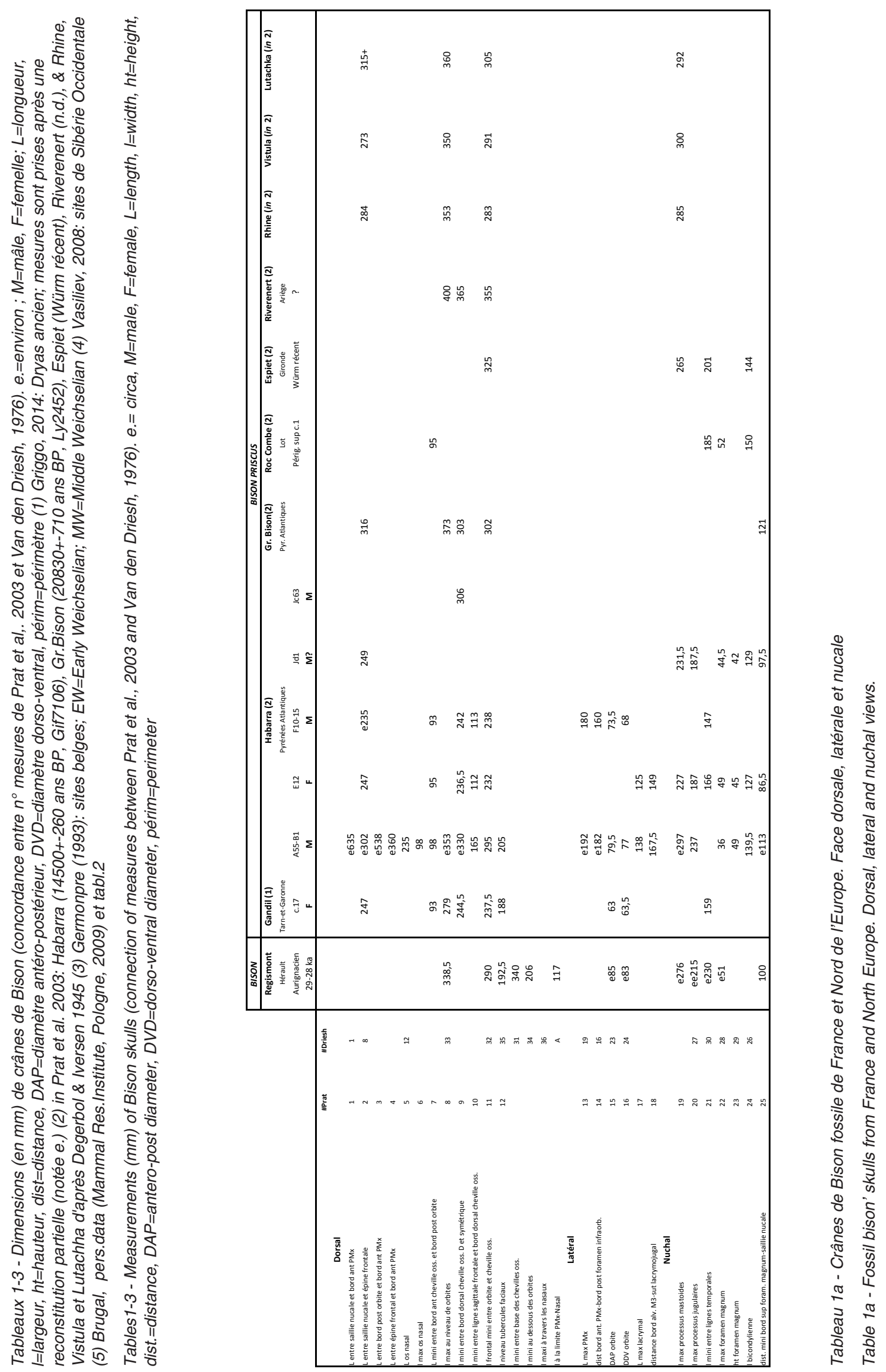


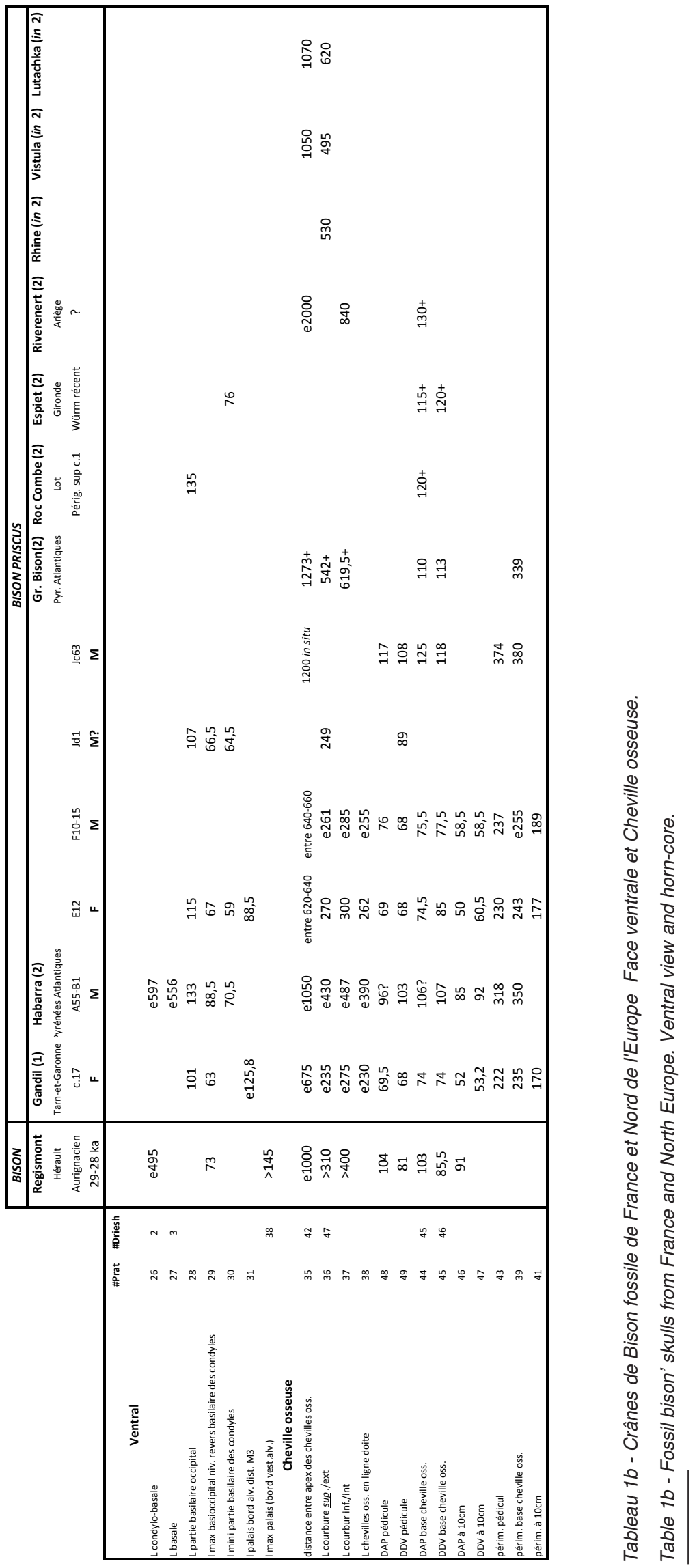




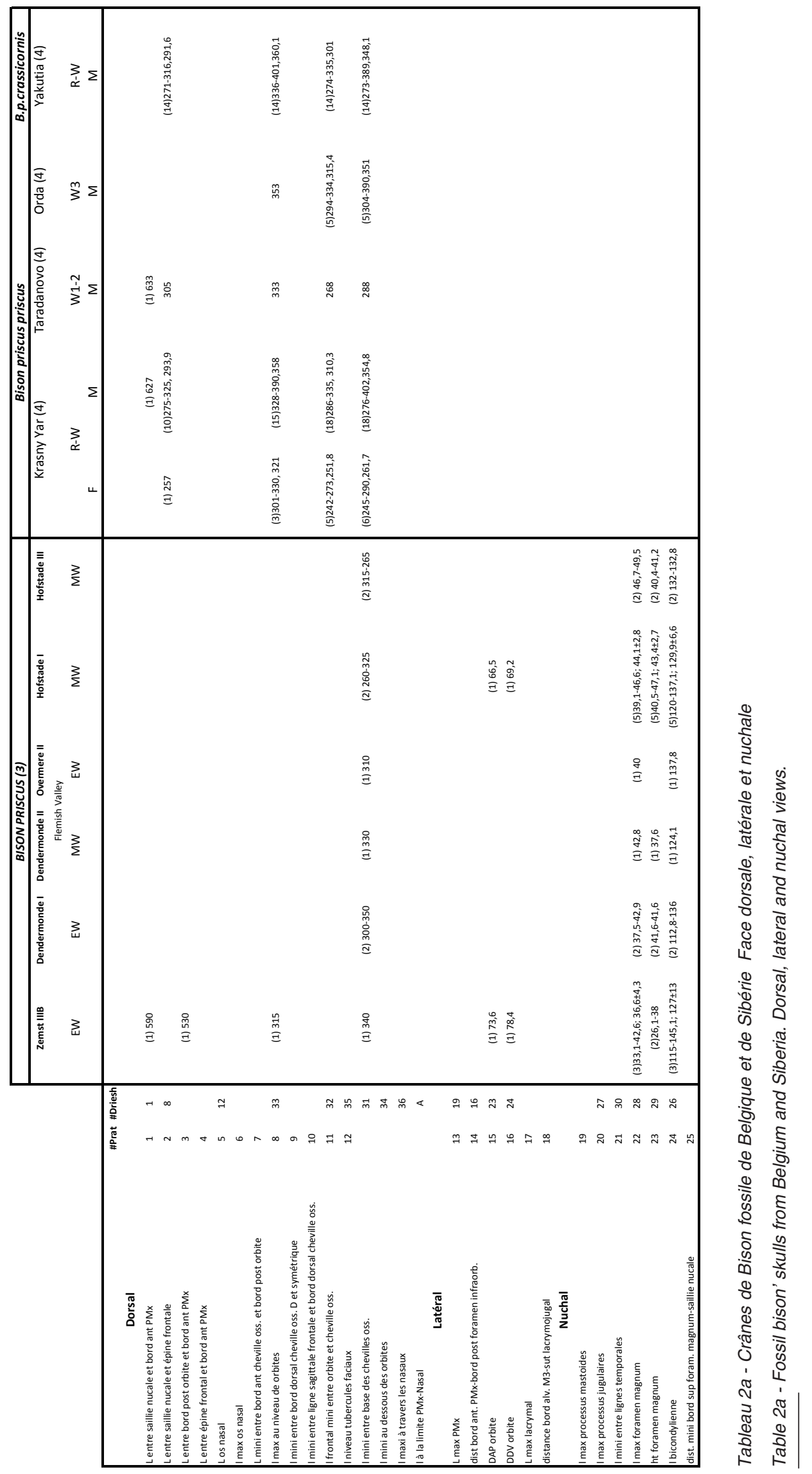




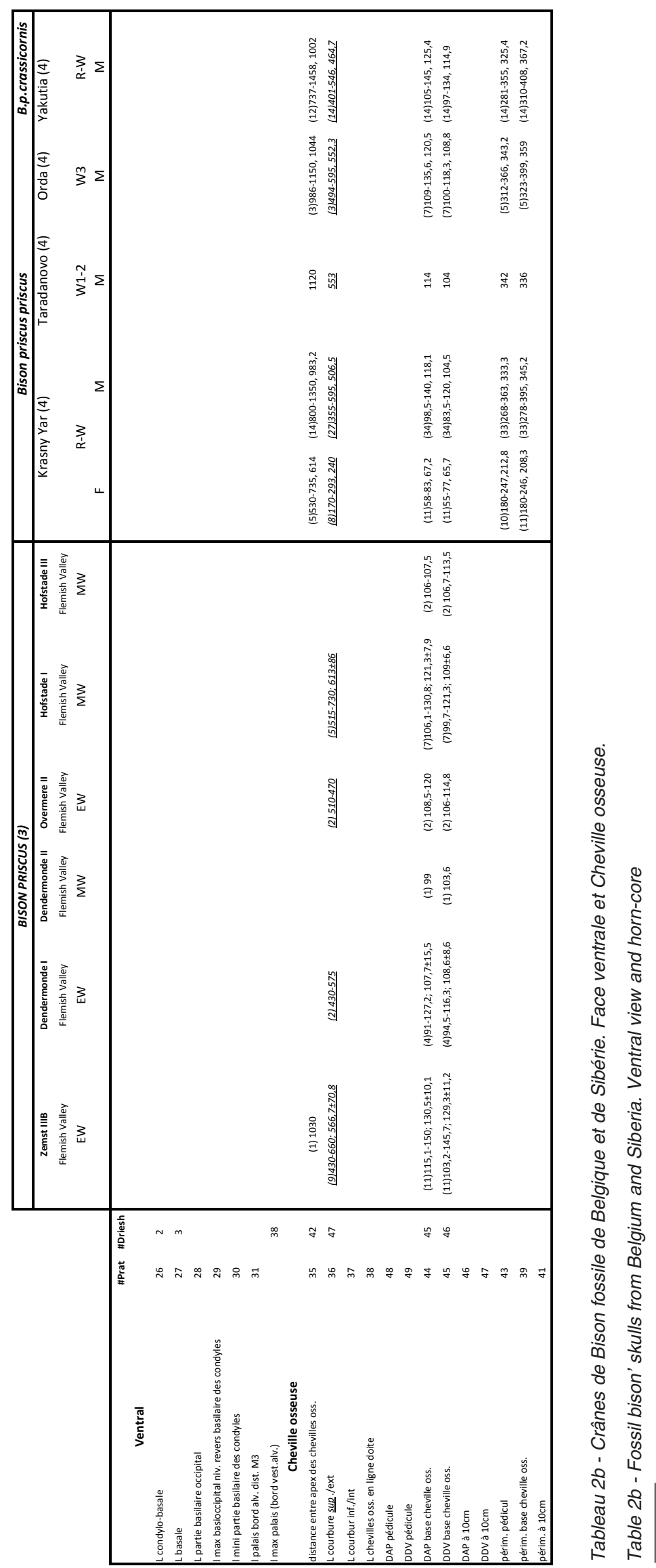




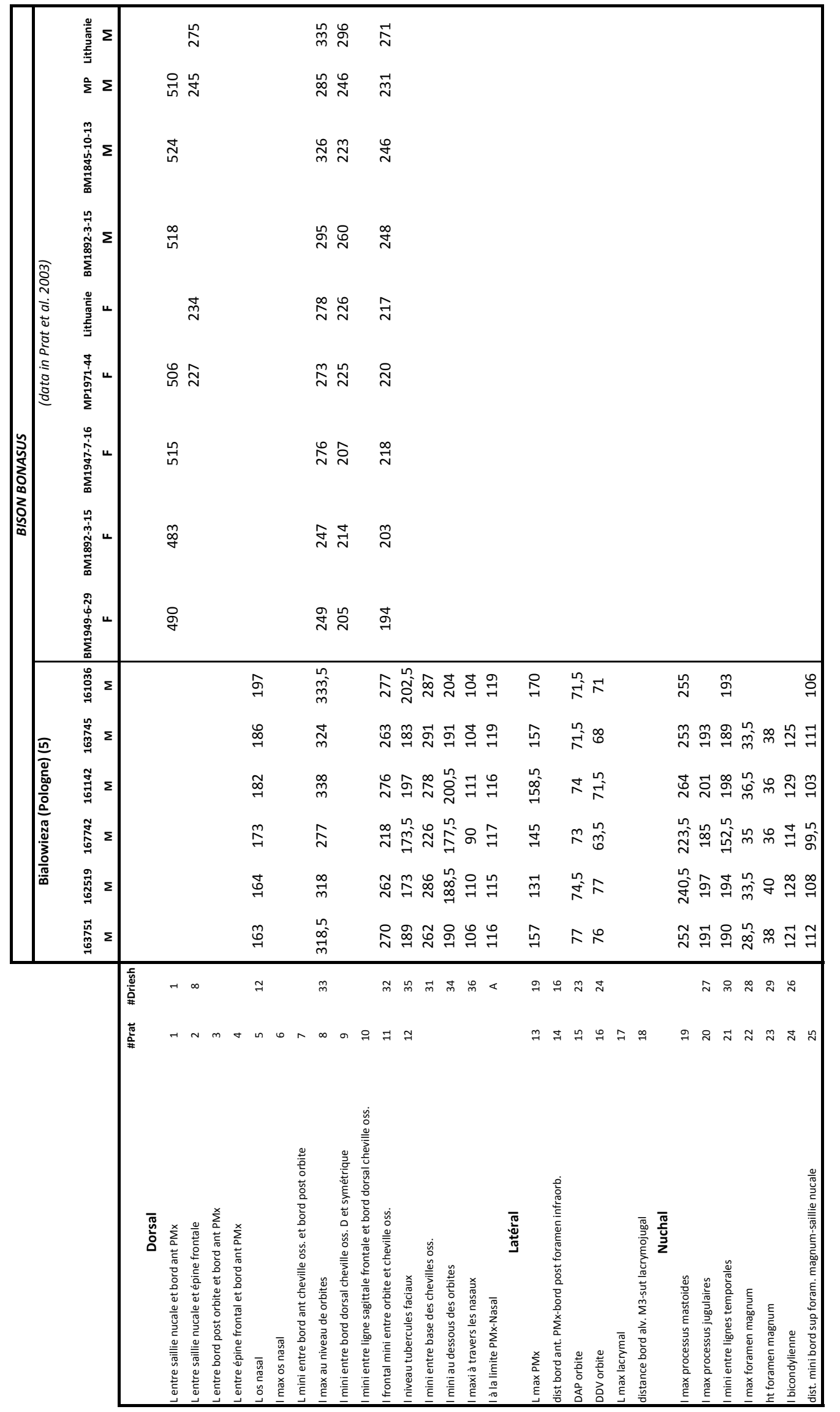




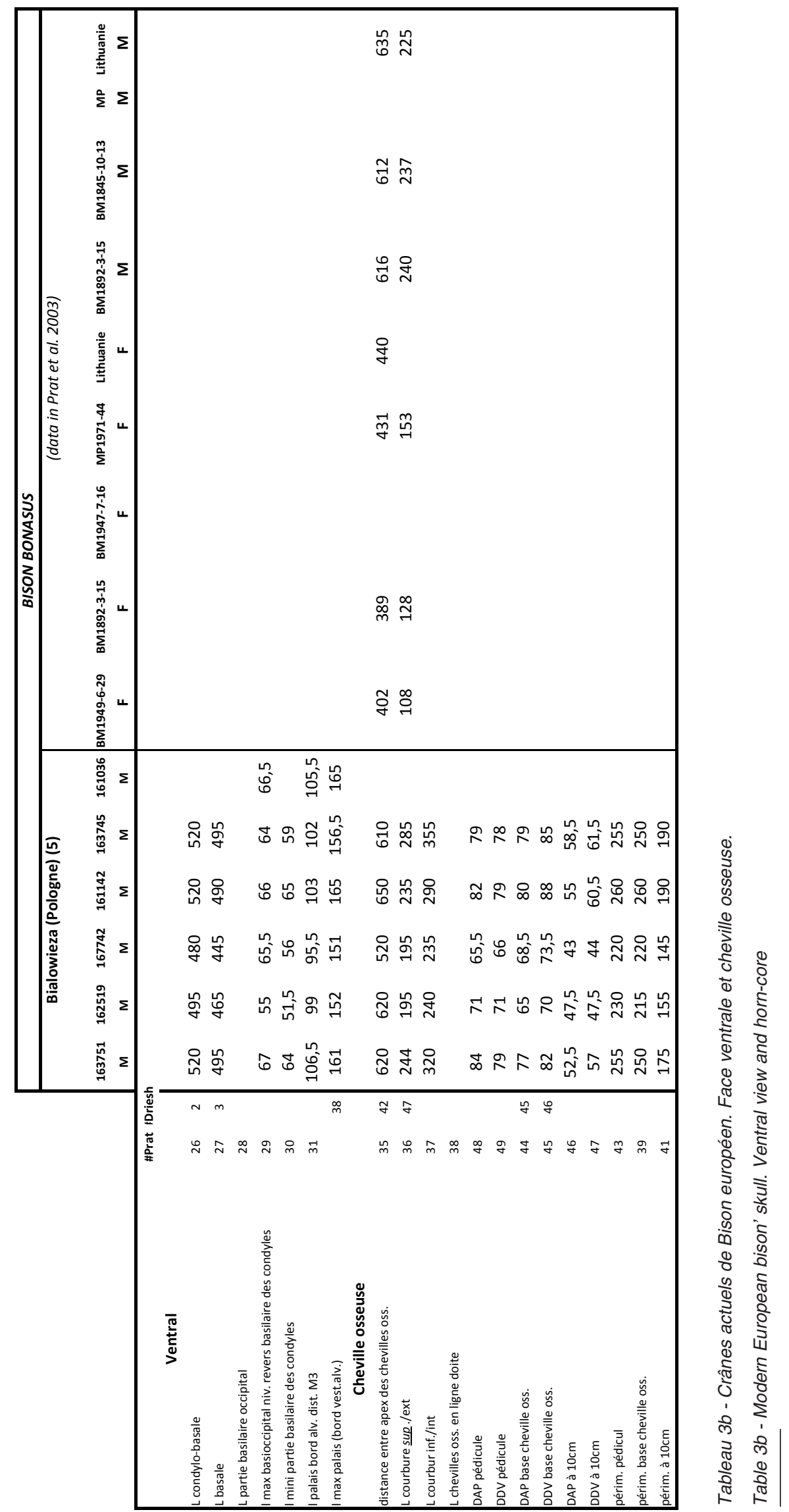




\begin{tabular}{|ccccc|}
\hline $\mathbf{n}^{\circ}$ & age (ans) & sexe & Poids (kg) & Ht garrot $(\mathbf{c m})$ \\
\hline 161036 & 12 & $\mathrm{M}$ & 790 & 187 \\
161142 & 9 & $\mathrm{M}$ & 740 & 177 \\
162519 & 5 & $\mathrm{M}$ & & \\
163745 & 7 & $\mathrm{M}$ & & \\
163751 & 6 & $\mathrm{M}$ & 650 & 180 \\
167742 & 4 & $\mathrm{M}$ & & \\
\hline
\end{tabular}

Tableau 4 - Données complémentaires sur l'échantillon de B.bonasus de Pologne (Mammal Research Institute, Bialowieza).

Table 4 - Complementary data of recent sample of Bison bonasus (Mammal Research Institute, Bialowieza, Poland).

\section{Conclusions}

Dans cette contribution, nous proposons l'histoire ancienne (paléo-histoire : Valentin 2008, 2011) d'un crâne découvert, et conservé, dans le contexte original d'un campement de plein air de chasseurs aurignaciens. Une histoire révélée par une analyse taphonomique et paléontologique qui permet de déterminer la nature de cette pièce et, au-delà, de proposer une évaluation à portée symbolique, ou a minima allégorique. Les exemples archéologiques autant qu'ethnographiques témoignent d'une certaine universalité dans ces pratiques chargées d'émotions et d'« aventures iconiques » (Otte 2016).

Les troupeaux de bison devaient trouver un biotope particulièrement adapté à leur vie dans les plaines du Languedoc sous le climat relativement frais du SIM3 et un paléoenvironnement ouvert. Ils constituaient l'un des gibiers préférentiels des groupes humains préhistoriques (Brugal et al. 1999), et un animal majeur pour eux ainsi qu'ils l'ont montré dans leur ornementation de certaines grottes (par ex. Aurignacien de la grotte Chauvet dans l'Ardèche, Chirica 2006) ; le bison est un élément quasi constant du bestiaire des hommes du Paléolithique supérieur, déterminant dans sa dimension socioéconomique. Abondance et permanence de ce gibier de choix dans ces plaines, forment sans doute l'un des facteurs clefs de l'installation des Aurignaciens en ces lieux. L'individu mâle auquel appartenait la tête-trophée étudiée devait représenter un animal pesant entre 800 et $1000 \mathrm{~kg}$ (voir tabl. 4 pour les sujets modernes), ce qui signifie près de $320-400 \mathrm{~kg}$ de viande, graisse et moelle (e. $40 \%$ du poids vivant). Une telle masse de viande pouvait alimenter un groupe important (quelques dizaines de personnes) durant plusieurs semaines. Avec le problème de la dégradation naturelle de la viande, des techniques de stockage et de conservation pourraient être suspectées ; ce

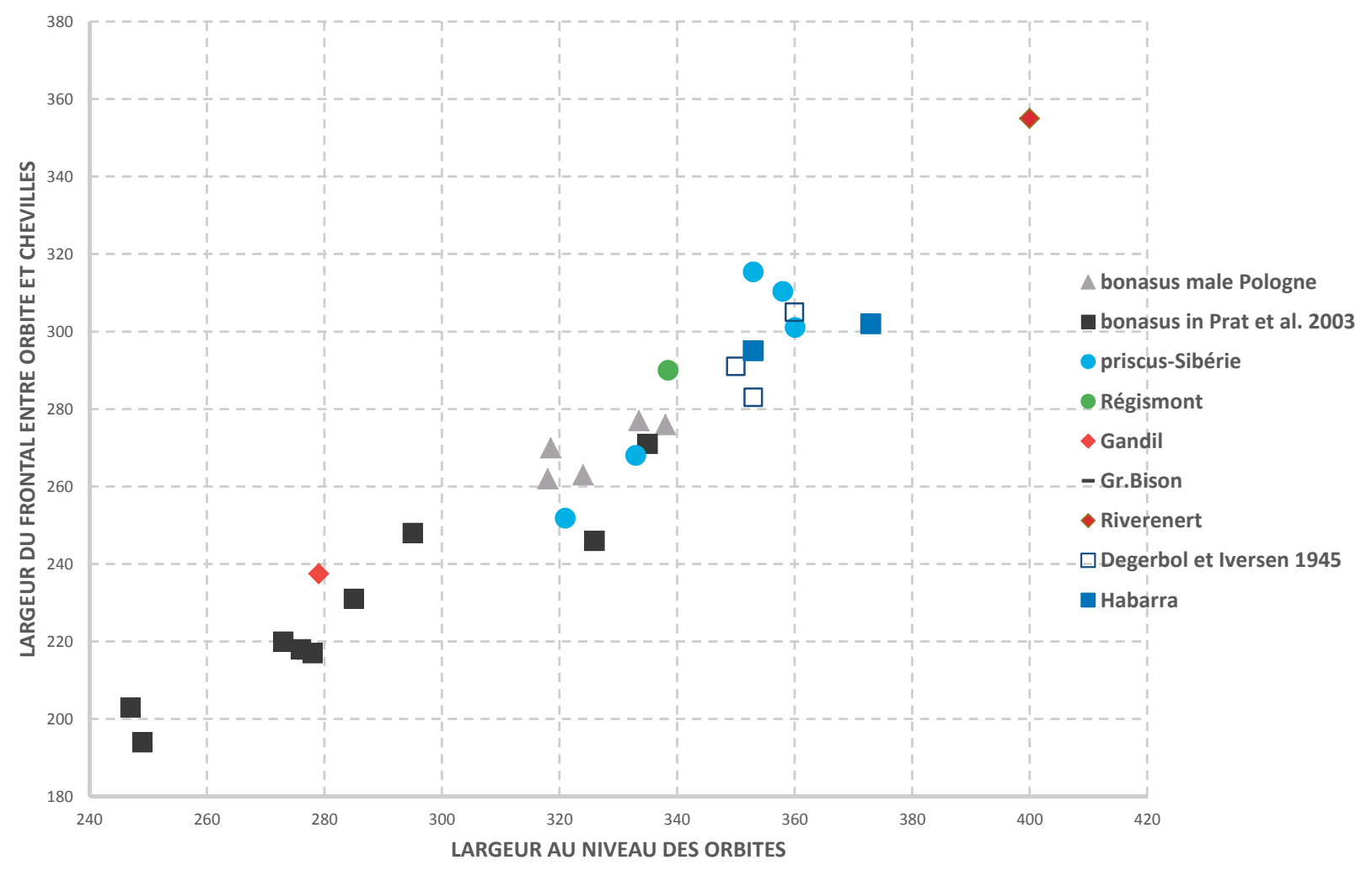

Figure 4 - Diagramme de dispersion des bisons selon des mesures crâniennes, mesures 32 et 33 (cf. tabl.1-3).

Figure 4 - Scatter-diagram of cranial measurements of bison, mes. 32 and 33 (see tabl. 1-3). 


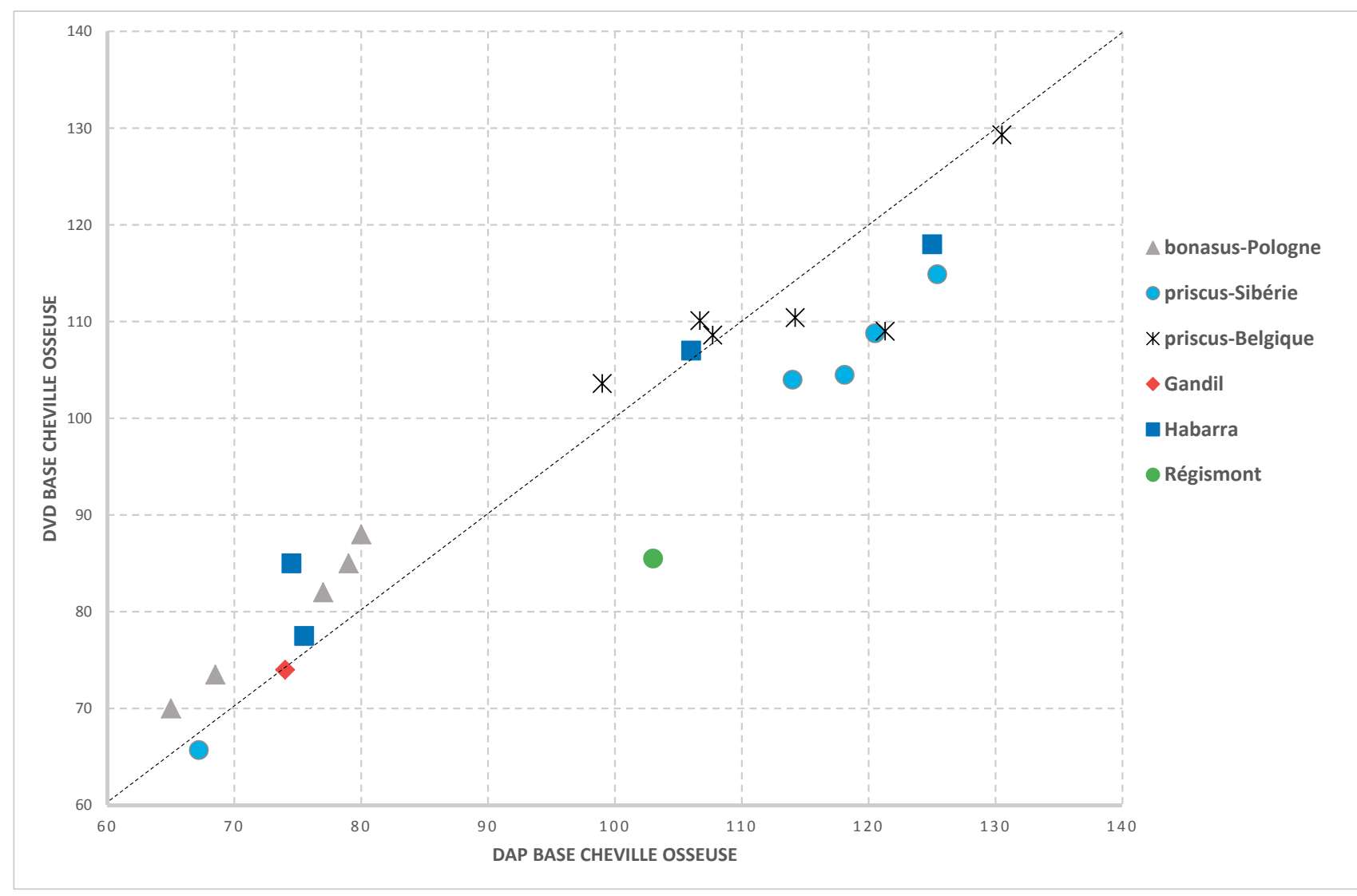

Figure 5 - Diagramme de dispersion des bisons selon des mesures sur les chevilles osseuses, mesures 45 et 46 (cf. tabl. 1-3). La ligne correspond a une section circulaire (rapport de $100 \%$ ).

Figure 5 - Scatter-diagram of horn-core measurements of bison, mes. 45 and 45 (see tabl. 1-3). The line indicate a ratio of $100 \%$ with a circular section.

que nous mettrions volontiers en rapport avec le grand nombre de foyers trouvés dans l'emprise du campement ou, tout au moins, de la zone fouillée, accompagnés d'un travail intensif des peaux de ces animaux (cf. supra). On renverra à la synthèse archéologique et ethnographique de J. Speth (2017) sur l'ensemble des aspects (social, économique, technologique, nutritionnel,...) des groupes humains dans l'exploitation et la consommation de bison. Dans le cadre d'acquisition de gibiers tels que les bisons, on peut ajouter qu'une tête devait peser plusieurs dizaines de kilos ; son abandon à proximité de ce qui apparaît comme un espace de boucherie primaire corrobore le fait que l'abattage de cet animal a dû se faire à proximité immédiate du campement, dans un environnement proche - si tant est que ce ne soit pas lui qui ait déterminé tout simplement son implantation.
Enfin, les nombreuses représentations de bisons dans l'art paléolithique (à corne longues ou plus courtes) rehaussent d'autant, à notre opinion, l'interprétation symbolique qui est proposée ici. Le bison s'inscrit dans un éventail de rôles fonctionnels : alimentaire, technique (nombreux sousproduits), symbolique ou spirituel. Les Bovidés, avec les Equidés, forment une « di-ade » immuable depuis les dessins et peintures paléolithiques, à fondement cynégétique, ou plus, jusqu'à ce même couple soufflant et offrant leurs chaleurs, et plus, à une divinité (crèche).

Histoires d'«hécatombes ${ }^{3} » \ldots$

\section{Remerciements}

Je tiens à chaleureusement remercier F. Bon et R. Mensan, responsables des opérations de fouilles programmées de

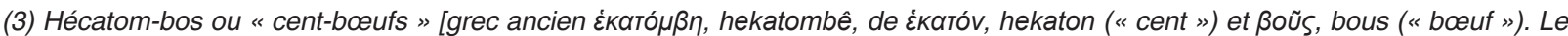
nombre de bêtes égorgées lors de sacrifices; terme utilisé ici en clin d'œil et de nombreux exemples de tuerie en masse (chasse collective) de bovinés (bison, bœuf) sont mentionnés, depuis le Paléolithique jusqu'à nos jours (Brugal et al. 1999, 2013), particulièrement dans des "sociétés à bison", dont ils constituaient une des ressources principales. La valeur symbolique des grands Bovidés est largement reconnue dans des sociétés très différentes de l'hémisphère Nord, du Pléistocène et de l'Holocène. 
Régismont-le-Haut, ainsi que toute leur équipe, en particulier S. Costamagno et D. Kuntz, de m'avoir invité à étudier cette pièce particulièrement intéressante et qui se prête à quelques jeux d'hypothèses originales. La mission en Pologne (pgm. Franco-Polonais, MAE, 2007) a été possible grâce à $\mathrm{Ph}$. Fosse que je salue ici. Je remercie J. Speth des échanges transcontinentaux sur notre (ou un de nos) animal favori, le bison. Merci à $F$. Delpech et J.-C. Castel de leur lecture attentive, et nécessaire.

\section{Références bibliographiques}

ANDERSON L., LEJAY M., BON F., MENSAN R., BRUGAL J.-PH., COSTAMAGNO S., HECKEL C., ARAUJO M., MÉNARD M., PRADEAU J-V., SALOMON H., SELLAMI F., BARSHAY-SZMIDT C. (à par.) - Insights into Aurignacian daily life and camp organization: the open-air site of Régismont-le-Haut. Quaternary International.

BEMENT L.C. 2003 - Constructing the Cooper model of Folsom bison kills on the southern plains. Great Plains Research 13 (Spring 2003): 27-41.

BOHLKEN H. 1958 - Vergleichende Untersuchungen an Wildrindern (Tribu Bovini Simpson 1945). Zool.Jahrb.Abt., $68: 113-202$.

BON F., MENSAN R., ET COLL. DE ARAUJO IGREJA M., COSTAMAGNO S., GARDĖRE P., MÉNARD C., SELLAMI F., SZMIDT C., THÉRY-PARISOT I. 2007 - Le site de plein air de Régismont-le-Haut : une halte aurignacienne dans les plaines du Languedoc. In : Qui est l'Aurignacien ? Aurignac, Editions Musée-forum, cahier 3, p. 53-71.

BON F., MENSAN R. ET ARAUJO IGREJA M., BRUGAL J.-PH., COSTAMAGNO S., GARDĖRE PH., KUNTZ D., SELLAMI F., SZMIDT C., THÉRY-PARISOT I. 2008 Poilhes, Régismont-le-Haut. In : Bilan scientifique de la région Languedoc- Roussillon 2005. Ministère de la Culture et de la Communication, p. 161-163.

BON F., MENSAN R., ANDERSON L., LEJAY M., ALEXIS M., BRUGAL J.-Ph., COSTAMAGNO S., HECKEL C., IGREJA M., PRADEAU J.V., QUÉNÉA K., SALOMON H., SELLAMI F., SZMIDT C., THERYPARISOT I. soumis - Régismont-le-Haut, fenêtre ouverte sur l'organisation d'un campement résidentiel aurignacien. Actes de la table ronde Habitat au Paléolithique supérieur, C. Montoya, P. Bodu, C. Paris (org.), Congr. Préh. De France, Amiens 2016.

BRUGAL J.-P. 1994-1995 - Le Bison (Bovinae, Artiodactyla) du gisement Pléistocène moyen ancien de Durfort (Gard, France). Bull.Mus.nat.Hist.Nat., Paris, $4^{\circ}$ sér. 16, sect.C, $n^{\circ} 2-4: 349-381$.

BRUGAL J.P. 1999 - Étude des populations de grands Bovidés européens : intérêt pour la connaissance des comportements humains au Paléolithique. In : Le Bison, gibier et moyen de subsistance des hommes du paléolithique aux paléoindiens des grandes plaines, J.-Ph. Brugal,
F. David, J. Enloe, J. Jaubert (dir.), (op.cit.) : 85-103.

BRUGAL J.P., DAVID F., ENLOE J., JAUBERT J., (dir.) 1999 - Le Bison : gibier et moyen de subsistance des hommes du Paléolithique aux Paléoindiens des grandes plaines. APDCA, Antibes, 562 p. (Actes du Colloque international, Toulouse, 6-10 juin 1995).

BRUGAL J.P., FOSSE P. 2005 - Les grands bovidés (Bison cf. schoetensackı) du site pléistocène moyen de La Vayssière (Aveyron, France). Actes du Coll. Les Ongulés Holarctiques du Pliocène et du Pléistocène, E.CrégutBonnoure (ss.la dir.), Quaternaire, H.S. $n^{\circ} 2$ : 75-80.

BRUGAL J.P. (coord), BEAUVAL C., COUMONT M.P., FOURNIER J., GERBE M., GRIGGO C. 2013 - Stratégies d'exploitation des ressources carnées au Paléolithique moyen en Quercy. In : Modalité d'occupation et exploitation des milieux au Paléolithique dans le Sud-Ouest de la France : l'exemple du Quercy. Paleo, supplément $\mathrm{n}^{\circ} 4$ (Actes de la session C67, XVème Congrès mondial de l'UISPP, Lisbonne, sept. 2006) : 213-229.

CHIRICA V. 2006 - Opinions concernant l'art et les religions paléolithiques sur la Base d'une grande découverte. Archeologia Moldovei, XXIX: 185-191.

COOPER A., TURNEY C., HUGHEN K.A., BROOK B.W., McDONALD H.G., BRADSHAW C.J.A. 2015 - Abrupt warming events drove Late Pleistocene Holarctic megafaunal turnover. Science, 349 (6248): 602-606.

CROUZEL F., MANENC A., REVEL J-C. 1982 - Squelettes de Bison priscus Bojanus ssp gigas Flerov, 1969 dans une grotte ariégeoise. Bulletin de la Société d'Histoire naturelle de Toulouse, $118: 71-100$.

DEGERBØL M., IVERSEN J. 1945 - The Bison in Denmark. A zoological and geological investigation of the finds in Danish Pleistocene deposits. København: Danm.Geol.undersøgelse, 62p. (Publ.73).

DRIESCH A. von den 1976 - A guide to the measurement of animal bones from archaeological sites. Peabody Museum, Harvard University Cambridge, Bull. $n^{\circ} 1,136 \mathrm{p}$.

EMPEL W. 1962 - Morphologie des schadels von Bison bonasus (Linnaeus 1758). Acta Theriologica, 6(4): 53-111+ph.

GAMBERI ALMENDRA DE CARVALHO L., ARGANT A., ARGANT J., BARTH P., BOUDADI-MALIGNE M., BOULBES N., BRUGAL J.-P., CARAMELLI D., CONDEMI S., CRÉGUT E., DEBARD E., ERRERA M.G.L., FARRE B., FAURE M., FERNANDEZ P., GEIGL E.-M., GUÉRIN C., HARTER S., JEANNET M., LATEUR N., MALLYE J.-B., MARTIN S., MONNEY J., ROMAN C., ROUSSELIĖRES F., SABAUT M., VALLI A. 2011- L'aven de l'Arquet - Barjac (30) : étude d'un aven piège. Ardèche Archéologie, 28 : 3-10.

GARRAIT-BOURRIER A. 2006 - Spiritualité et Fois Amérindiennes. Résurgence d'une identité perdue. Cercles 15 : 98-95. 
GERAADS D. 1992 - Phylogenetic analysis of the tribe Bovini (Mammalia, Artiodactyla). Zool. J. Linn. Soc. Lond. 104, 193-207.

GERAADS D. 1992 - Phylogenetic analysis of the tribe Bovini (Mammalia, Artiodactyla). Zool. J. Linn. Soc. Lond., 104, 193-207.

GERMONPRÉ M. 1993 - Osteometric data on Late Pleistocene mammals from the Flemish Valley, Belgium. Documents de travail de l'Institut Royal de Sciences Naturelles Belge, $n^{\circ} 72: 5-135$.

GRIGGO C. 2014 - La faune magdalénienne de l'abri Gandil à Bruniquel (Tarn-et-Garonne). Etudes paléontologique, taphonomique et archéozoologique. In : L'abri Gandil à Bruniquel (Tarn-et-Garonne) : un campement magdalénien du temps de Lascaux. E.Ladier (dir.), Préhistoire du Sud-Ouest, suppl. 13 : 49-71.

GROMOVA V.I., 1932 - Uber den typus des Bison priscus Boj. Zool.Anz., 99 : 207-221.

GROMOVA V.I. 1935 - Die vorweltliche Wisent (Bison priscus Bojanus) in USSR.

Trav.Inst.Paleozool.Acad.Sci.URSS, 2 (2/3):77-204.

GROVES C.P. 1981 - Systematic relationship in the Bovini (Artiodactyla, Bovidae). Z.Zool.Syst.Evolutionforsch., 19/4 : 264-278.

HASSANIN A., AN J., ROPIQUET A., THANH NGUYEN T., COULOUX A. 2013 - Combining multiple autosomal introns for studying shallow phylogeny and taxonomy of Laurasiatherian mammals: Application to the tribe Bovini (Cetartiodactyla, Bovidae). Molecular Phylogenetics and Evolution, 66:766-775.

HILL A. 1979 - Disarticulation and Scattering of Mammal Skeletons. Paleobiology, (5, 3): 261-274.

HILL A., BEHRENSMEYER A.K. 1984 - Disarticulation Patterns of Some Modern East African Mammals. Paleobiology, (10, 3): 366-376.

HILL A., BEHRENSMEYER A.K. 1985 - Natural Disarticulation and Bison Butchery. American Antiquity, (50, 1): $141-145$.

KUNTZ D. 2006 - Notes sur la restauration du crâne de Bison du secteur S67. Pp.51-55. In : Bon F. et al. Régismont-le-Haut (Poilhes, Hérault). Campagne de fouilles d'un habitat de plein air aurignacien. Rapport d'opération de fouille programmée, SRA Languedoc-Roussillon, $68 \mathrm{p}$.

FLEROW C.C. 1975 - Die Bison-Reste aus den Travertinen von Weimar-Ehringsdorf.. Abh.Zentr.Geol.Inst., 23 : 171-199.

FLEROW C.C. 1976 - Die fossilen Bisonreste von Taubach und ihre Stellung in der Entwicklungs geschichte der Gattung Bison in Europa. Quartär Paläontologie, 2 : 179-208.
FLEROW C.C. 1979 - Systematics and Evolution. In: V. E. Sokolov (Dir.), European Bison -Morphology, systematics, evolution, ecology (en russe), Moscou, Nauka, p. 9-127 (USSR Ac. of Sc.).

FRISON G.C. 1971 - The Buffalo Pound in North-Western Plains Prehistory: Site 48 CA 302, Wyoming. American Antiquity, 36(1): 77-91.

MASSILANI D., GUIMARAES S., BRUGAL J.P., BENNETT E.A.,TOKARSKA M., ARBOGAST R.A., BARYSHNIKOV G., BOESKOROV G., CASTEL J.C., DAVYDOV S., MADELAINE S., PUTELAT O., SPASSKAYA N., UERPMANN H.P., GRANGE T., GEIGL E.M. 2016 - Past climate changes, population dynamics and the origin of Bison in Europe. BMC Biology, 14: 93, DOI 10.1186/s12915-016-0317-7

McDONALD J.N. 1981 - North American Bison. Their classification and evolution. Berkeley: Univ. California, 316p.

NICHOLSON N., NICHOLSON S. 2007 - Late prehistoric ceremonial treatment of a bison skull in southwestern Manitoba. Plains Anthropologist, 52:203, 317-324, DOI: 1 0.1179/pan.2007.021

OTTE M. 2016 - Arts et pensée dans l'évolution humaine. C. R. Palevol, http://dx.doi.org/10.1016/j.crpv.2016.05.001

PALACIO P., BERTHONAUD V., GUÉRIN C., LAMBOURDIĖRE J., MAKSUD F., PHILIPPE M., PLAIRE D., STAFFORD T., MARSOLIERKERGOAT M.C., ELALOUF J.M. 2017 - Genome data on the extinct Bison Schoetensacki establish it as a sister species of the extant European bison (Bison bonasus) BMC Evolutionary Biology 17:48.DOI 10.1186/s12862-0170894-2.

PFEIFFER T. 1999 - Systematic relationship within the Bovini, with special reference to the fossil taxa Bos primigenius Bojanus and Bison priscus Bojanus. In : G.C. Weniger (dir), Archäologie und Biologie des Auerochsen. Neanderthal Museum, 1: 60-70.

PRAT F., DELPECH F., CANCEL N., GUADELLI J.L., SLOTT-MOLLER R. 2003 - Le Bison des steppes, Bison priscus Bojanus, 1827, de la grotte d'Habarra à Arudy (Pyrénées-Atlantiques). Paleo, cahier spécial n5: 1-102.

PILGRIM G.E. 1947 - The evolution of the buffaloes, oxen, sheep and goats. J. Linn. Soc. Lond., 279: 272-286.

REHER C.A., FRISON G.C. 1980 - The Vore site, 48ck302, A stratified buffalo jump in the Wyoming black hills. Plains Anthropologist, Memoir 16 (25): 221 p.

SALA B. 1987 - Bison schoetensacki Freud. from Isernia la Pineta (early Mid-Pleistocene, Italy) and revision of the European species of bison. Palaeontogr. Ital., 74: 113-170.

SOUBRIER J., GOWER G. CHEN K., RICHARDS S.M., LLAMAS B., MITCHELL K.J., HO S.Y.W., KOSINTSEV P., LEE M.Y., BARYSHNIKOV G., BOLLONGINO R., 
BOVER P., BURGER J., CHIVALL D., CREGUTBONNOURE E., DECKER J.E., DORONICHEV V.B., DOUKA K., FORDHAM D.A., FONTANA F., FRITZ C., GLIMMERVEEN J., GOLOVANOVA L.V., GROVES C., GUERRESCHI A., HAAK W., HIGHAM T., HOFMAN, KAMINSKA E., IMMEL A., JULIEN M.A., KRAUSE J., KROTOVA O., LANGBEIN F., LARSON G., ROHRLACH A., SCHEU A., SCHNABEL R.D., TAYLOR J.F., TOKARSKA M., TOSELLO G., PLICHT J.VAN DER, LOENEN A. VAN, VIGNE J.D., WOOLEY O., ORLANDO L., KOWALCZYK R., SHAPIRO B., COOPER A. 2016 - Early cave art and ancient DNA record the origin of European bison. Nature Communications, DOI: 10.1038/ncomms13158.

SOKOLOV V.E., (Dir.) 1979 - European Bison Morphology, systematics, evolution, ecology (en russe), Moscou, Nauka, (USSR Ac. of Sc.), 495p.

SPASSOV N., STOYTCHEV T. 2003 - On the origin of the wisent, Bison bonasus (Linnaeus, 1758): presence of the wisent in the upper Palaeolithic art of Eurasia. Advances in Vertebrate Paleontology « Hen to Panta », 125-130.

SPETH J.D. 2017 (ss-pr) - A New Look at Old Assumptions: Paleoindian Communal Bison Hunting, Mobility, and Stone Tool Technology. In : Manipulating Prey: Development of Large-Scale Kill Events Around the
Globe. K. Carlson and L. Bement (eds), University Press of Colorado, Boulder, CO: \& 8 .

TODD L.C., RAPSON D.J. 1999 - Formational analysis of bison bonebeds and interpretation of Paleoindian subsistence. In : Le Bison : gibier et moyen de subsistance des hommes du Paléolithique aux Paléoindiens des grandes plaines, (op.cit.): 479-499.

VALENTIN B. - 2008 - Jalons pour une paléohistoire des derniers chasseurs (VIVé-VIé millénaire avant J.C.). Publication de la Sorbonne. 329 p.

VALENTIN B. 2011 - Le Paléolithique. Presses Universitaires de France, «Que sais-je ?» n 3924.

VASILIEV S.K. 2008 - Late Pleistocene bison (Bison $p$. priscus Bojanus, 1827) from the southeastern part of Western Siberia. Archaeology Ethnology \& Anthropology of Eurasia 34/2: 34-56

WALL D.A., DAVIS S.K., READ B.M. 1992 - Phylogenetic relationships in the subfamily Bovinae (Mammalia: Artiodactyla) based on ribosomal DNA. J.Mamm., 73(2): 262-275. 